\title{
Contribuição ao estudo fitoquímico de Dorstenia multiformis Miquel (Moraceae) com abordagem em atividade antioxidante
}

\author{
Luciana Balestrin, ${ }^{1}$ Josiane F. Gaspari Dias, ${ }^{2}$ Obdúlio Gomes Miguel, ${ }^{* 2}$ Daniela S. G. \\ Dall'Stella, ${ }^{1}$ Marilis Dallarmi Miguel ${ }^{1}$ \\ 'Departamento de Farmácia, Laboratório de Farmacotécnica, Universidade Federal do Paraná, Av. Prefeito \\ Lothário Meissner 3400, 80210-170 Curitiba-PR, Brasil, \\ ${ }^{2}$ Departamento de Farmácia, Laboratório de Fitoquímica, Universidade Federal do Paraná, Av. Prefeito \\ Lothário Meissner 3400, 80210-170 Curitiba-PR, Brasil
}

\begin{abstract}
RESUMO: Conhecida como carapiá, a espécie Dorstenia multiformis Miquel, Moraceae, é largamente empregada na medicina popular contra doenças de pele. Neste trabalho determinouse a atividade antioxidante de substâncias isoladas, do extrato etanólico, e frações hexano, clorofórmio e acetato de etila. Todas as amostras testadas apresentaram atividade antioxidante, sendo que a fração clorofórmio foi a que apresentou maior atividade antioxidante.
\end{abstract}

Unitermos: Dorstenia multiformis, fitoquímica, atividade antioxidante, carapiá.

\begin{abstract}
Contribution to the phytochemical study of Dorstenia multiformis Miquel (Moraceae) with approach in antioxidant activity". The species Dorstenia multiformis Miquel, Moraceae, known as "carapiá", is largely employed in folk medicine against skin diseases. In this paper the antioxidant activity of isolated substances, from the ethanol extract, and hexane, chloroform and ethyl acetate fractions has been determined. All of the tested samples showed antioxidant activity, and the chloroform fraction was the one that presented the largest antioxidant activity.
\end{abstract}

Keywords: Dorstenia multiformis, phytochemistry, antioxidant activity, "carapiá".

\section{INTRODUÇÃO}

A família Moraceae apresenta aproximadamente 61 gêneros, com mais de 1000 espécies, e se encontra bem representada no Brasil, tanto por espécies indígenas, como por cultivadas. É freqüente, de um modo geral, nas regiões tropicais e subtropicais de todo o mundo, sendo menos comum em climas temperados (Joly, 1975; Cronquist, 1981).

Segundo Abegaz et al. (2002), o gênero Dorstenia possui muitas plantas que são utilizadas como antiofídicas, antiinfecciosas e anti-reumáticas.

Algumas espécies deste gênero são utilizadas na medicina humana, principalmente contra tosse e bronquite (Agra et al., 2007) e doenças de pele, pela presença de furanocumarinas (Cardoso et al., 2002) e compostos do tipo terpenóides têm sido relatados como os responsáveis pelo uso popular destas plantas como antiofídicos (Vilegas et al., 1997).

Diversas substâncias foram isoladas do gênero Dorstenia, incluindo: psoraleno e bergapteno (Bauer \& Noll, 1986); ácidos graxos, triterpenos pentacíclicos, esteróides e furanocumarinas (Vilegas et al., 1992); butirospermol (Tsopmo et al., 1998); furanocumarinas (psoraleno, bergapteno, pimpinelina e isopimpinelina) (Cardoso et al., 2002); catequina e epicatequina (Caceres et al., 2001); um análogo do psoraleno, denominado dorstenina (Lopes et al., 2001); psoraleno, bergapteno, beta-sitosterol e seus derivados D-glucopiranosídicos (Abegaz et al., 2002); dorstenona (Tsopmo et al., 1998); flavonóides prenilados (Abegaz et al., 2002 e Ngadjui et al., 2002) e siriogenina (Casagrande et al., 1974), entre outros.

Os dados farmacológicos sobre o gênero Dorstenia são escassos, mas sabe-se que todas as espécies do gênero conhecidas como carapiá são dotadas de qualidades medicinais, entre elas podem-se citar: atividade analgésica e/ou antiinflamatória (Ruppelt et al.,1991; Abegaz et al., 2002; Falcão et al., 2005), atividade antileishmania (Iwu et al., 1992; Abegaz et al., 2002), atividade antioxidante (Abegaz et al., 2002), atividade citotóxica (Abegaz et al., 2002), atividade giardicida (Amaral et al., 2006), efeitos antihipertensivos e diminuição nos níveis de colesterol e insulina (Dimo et al., 2001).

\section{MATERIAL E MÉTODOS}

\section{Obtenção do material vegetal}

A espécie Dorstenia multiformis foi fornecida pela indústria farmacêutica "As Ervas Curam", em 
março de 2004. A coleta foi efetuada na Fazenda Capão Grande, situada no município de Jataí, estado de Goiás, em janeiro de 2004. Amostras da planta inteira foram enviadas ao Museu Botânico Municipal de Curitiba, para identificação botânica pelo botânico Dr. Gerdt Hatchbach do Museu Botânico Municipal (MBM) de Curitiba/PR, e encontra-se registrada neste museu sob o número 37440.

\section{Análise fitoquímica}

Foram submetidos $5000 \mathrm{~g}$ do material vegetal à extração com etanol absoluto, em aparelho de Soxhlet. O extrato obtido foi filtrado e concentrado em evaporador rotatório sob pressão reduzida, a temperatura de aproximadamente $40{ }^{\circ} \mathrm{C}$ e encaminhado ao freezer. Após 24 horas, o extrato concentrado foi filtrado a vácuo, obtendo-se o extrato etanólico bruto e o resíduo correspondente, os quais foram armazenados separadamente.

O resíduo resultante da filtração do extrato concentrado foi lavado com acetona, obtendo-se duas porções, uma solúvel em acetona e outra não solúvel em acetona. Da porção solúvel em acetona fez-se cromatografia em coluna utilizando-se como eluentes hexano e acetato de etila com variação de $10 \%$ no gradiente. Desta coluna isolaram-se dois compostos denominados CRS1 (753 mg) e CRS2 (52,6 mg). Estes compostos foram analisados por CG-EM, RMN ${ }^{13} \mathrm{C}$ e $\mathrm{RMN}^{1} \mathrm{H}$, os quais revelaram a presença de psoraleno e bergapteno no composto CRS1 e $\beta$-amirina e $\alpha$-amirina no composto CRS2.

$\mathrm{O}$ extrato bruto etanólico foi fracionado em aparelho de Soxhlet modificado (Carvalho, 2001), por meio de partição líquido-líquido utilizando-se solventes de polaridade crescente (hexano, clorofórmio e acetato de etila). Esse procedimento resultou nas frações hexano, clorofórmio e acetato de etila. Os valores do teor de sólidos do extrato bruto etanólico e das frações obtidas de D. multiformis encontram-se na Tabela 1.

Para RMN ${ }^{13} \mathrm{Ce}{ }^{1} \mathrm{Hutilizou}$-se espectrofotômetro Brucker $^{\circledR}$ modelo AC 200 em 50 MHZ. O solvente utilizado na espectroscopia de RMN foi clorofórmio deuterado $\left(\mathrm{CDCl}_{3}\right)$ e como padrão de referência interna utilizou-se tetrametilsilano. Para Cromatografia Gasosa acoplada em massa utilizou-se cromatográfo gasoso 6890 da Agilent ${ }^{\circledR}$ equipado com coluna HP5 (5\% fenil e $95 \%$ dimetilpolisiloxano) de $0,25 \mathrm{~mm}$ de diâmetro interno por $15 \mathrm{~cm}$ de comprimento, e espectrômetro de massa 5973 da Agilent ${ }^{\circledR}$. A identificação dos constituintes foi feita por comparação do espectro de massa do referido constituinte, com os espectros de massa existentes no banco de dados do equipamento (DATABASE/ NIST98).

\section{Avaliação da atividade antioxidante pelo método de complexação do fosfomolibdênio}

Este método, descrito por Prieto (1999), fundamenta-se na redução do molibdênio (VI) a molibdênio (V) ocorrida em presença de determinadas substâncias com capacidade antioxidante, com formação de um complexo verde entre fosfato/molibdênio $(\mathrm{V})$, em pHácido, o qualé determinado espectrofotometricamente a $695 \mathrm{~nm}$.

O complexo fosfomolibdênico, formado pela reação da solução de $\mathrm{Na}_{3} \mathrm{PO}_{4}(28 \mathrm{~mL}, 0,1 \mathrm{~mol} / \mathrm{L}) \mathrm{com}$ solução de $\left(\mathrm{NH}_{4}\right)_{6} \mathrm{Mo}_{7} \mathrm{O}_{24} \cdot 4 \mathrm{H}_{2} \mathrm{O}(12 \mathrm{~mL}, 0,03 \mathrm{~mol} / \mathrm{L}) \mathrm{e}$ solução de $\mathrm{H}_{2} \mathrm{SO}_{4}(20 \mathrm{~mL}, 3 \mathrm{~mol} / \mathrm{L})$, em meio aquoso. $\mathrm{O}$ volume final é ajustado com água destilada para 100 $\mathrm{mL}$, e a coloração amarela, torna-se verde à medida que se reduz.

Para o preparo do complexo fosfomolibdênico, todos os reativos foram transferidos para um balão volumétrico e o volume foi completado para $100 \mathrm{~mL}$ com água destilada.

O extrato e respectivas frações foram levados à secura em banho-maria $\left(40^{\circ} \mathrm{C}\right)$, e a partir do material seco, bem como das substâncias isoladas, prepararam-se soluções aquosas com concentração final de $200 \mu \mathrm{g} / \mathrm{mL}$, utilizando-se uma quantidade mínima de metanol para dissolver as amostras, quando necessário. Destas, foram tomados $0,3 \mathrm{ml}$ e adicionado $1 \mathrm{~mL}$ de solução reagente do complexo fosfomolibdênio. Os tubos foram fechados e mantidos em banho-maria a $95{ }^{\circ} \mathrm{C}$ por 90 min. Após resfriamento, procedeu-se a leitura a $695 \mathrm{~nm}$, em um espectrofotômetro UV-1601 Shimadzu ${ }^{\circledR}$, para obtenção das absorvâncias, usando-se água destilada como branco. A capacidade antioxidante das amostras foi expressa em relação à vitamina $\mathrm{C}(200 \mu \mathrm{g} / \mathrm{mL})$ usada como padrão, e rutina $(200 \mu \mathrm{g} / \mathrm{mL})$ cuja atividade antioxidante de referência foi considerada 1,00. A vitamina $\mathrm{C}$ é um dos antioxidantes biológicos mais eficazes e mais conhecidos (Youngson, 1996).

Para este ensaio utilizaram-se as seguintes amostras em duplicata: extrato etanólico bruto, frações hexano, clorofórmio e acetato de etila, e os compostos isolados CRS1 e CRS2.

Os resultados da avaliação da atividade antioxidante foram expressos na forma de atividade antioxidante relativa AAR\% (ác. ascórbico) e AAR\%(rutina). Os cálculos foram estabelecidos por meio das equações:

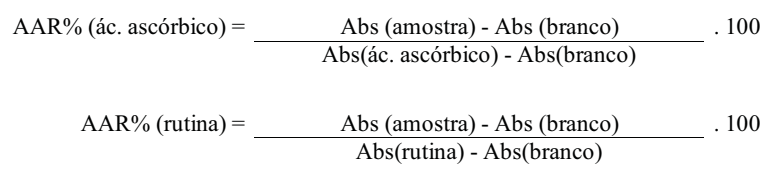

Avaliaçãodaatividadeantioxidanteporcromatografia de camada delgada 
Tabela 1. Teor de sólidos do extrato bruto etanólico e frações de Dorstenia multiformis Miquel.

\begin{tabular}{l|c|c}
\hline \multicolumn{1}{c|}{ Extrato/fração } & Resíduo seco & $\begin{array}{c}\text { Rendimento em relação à massa de 5000 g de } \\
\text { vegetal seco }\end{array}$ \\
\hline Extrato etanólico bruto & $78,5 \mathrm{mg} / \mathrm{mL}$ & $7,22 \mathrm{~g} \%$ \\
Fração hexano & $38,0 \mathrm{mg} / \mathrm{mL}$ & $3,50 \mathrm{~g} \%$ \\
Fração clorofórmio & $46,7 \mathrm{mg} / \mathrm{mL}$ & $4,30 \mathrm{~g} \%$ \\
Fração acetato de etila & $14,5 \mathrm{mg} / \mathrm{mL}$ & $1,33 \mathrm{~g} \%$ \\
\hline
\end{tabular}

Tabela 2. Deslocamentos em ppm do espectro de $\mathrm{RMN}{ }^{13} \mathrm{C}$ e ${ }^{1} \mathrm{H}$ para substâncias isoladas da amostra CRS1.

\begin{tabular}{|c|c|c|c|c|c|c|c|}
\hline \multicolumn{4}{|c|}{ Psoraleno } & \multicolumn{4}{|c|}{ Bergapteno } \\
\hline Carbono & Tipo & $\begin{array}{c}\text { Absorção } \\
\text { (ppm) }\end{array}$ & Proton & Carbono & Tipo & $\begin{array}{c}\text { Absorção } \\
\text { (ppm) }\end{array}$ & Proton \\
\hline 1 & & & & 1 & & & \\
\hline 2 & $\mathrm{CH}$ & 160,86 & & 2 & $\mathrm{CH}$ & 160,86 & \\
\hline 3 & $\mathrm{CH}$ & 112,18 & $\begin{array}{r}(5,58-5,78) \\
(1 \mathrm{H}, \mathrm{d}, \mathrm{H} 3)\end{array}$ & 3 & $\mathrm{CH}$ & 112,18 & $\begin{array}{c}(5,58-5,78) \\
(1 \mathrm{H}, \mathrm{d}, \mathrm{H} 3)\end{array}$ \\
\hline 4 & $\mathrm{CH}$ & 139,15 & $\begin{array}{c}(7,71-7,66) \\
(1 \mathrm{H}, \mathrm{d}, 9.2, \mathrm{H} 4)\end{array}$ & 4 & $\mathrm{CH}$ & 139,15 & $\begin{array}{c}(7,71-7,66) \\
(1 \mathrm{H}, \mathrm{d}, 9.2, \mathrm{H} 4)\end{array}$ \\
\hline 5 & $\mathrm{CH}$ & 144,62 & & 5 & $\mathrm{CH}$ & 144,62 & \\
\hline 6 & $\mathrm{C}$ & 104,9 & & 6 & $\mathrm{C}$ & 104,9 & \\
\hline 7 & $\mathrm{C}$ & 156,19 & & 7 & $\mathrm{C}$ & 156,19 & \\
\hline 8 & $\mathrm{CH}$ & 93,46 & $\begin{array}{c}(7,16) \\
(1 \mathrm{H}, \mathrm{s}, \mathrm{H} 8)\end{array}$ & 8 & $\mathrm{CH}$ & 93,46 & $\begin{array}{c}(7,16) \\
(1 \mathrm{H}, \mathrm{s}, \mathrm{H} 8)\end{array}$ \\
\hline 9 & $\mathrm{C}$ & 151,79 & & 9 & $\mathrm{C}$ & 151,79 & \\
\hline 10 & $\mathrm{C}$ & 112,18 & & 10 & $\mathrm{C}$ & 112,18 & \\
\hline $1 "$ & $\mathrm{CH}$ & 104,9 & 6,40 (1H, d, H2”) & $1 "$ & $\mathrm{CH}$ & 104,9 & 6,40 (1H, d, H2”) \\
\hline & & & & $2 "$ & $\mathrm{CH}$ & 143,9 & $(6,62-6,59)(1 \mathrm{H}, \mathrm{d}, \mathrm{H} 2 ")$ \\
\hline & & & & & $\mathrm{OCH}_{3}$ & 59,88 & $3,83(3 \mathrm{H}, \mathrm{s}, \mathrm{J} 9,34)$ \\
\hline
\end{tabular}

Tabela 3. Valores de deslocamento químico de $\mathrm{RMN}$ de ${ }^{13} \mathrm{C} / \mathrm{DEPT} /{ }^{1} \mathrm{H}\left(\mathrm{CDCl}_{3}\right.$, TMS, $\left.\Delta\right)$ para a amostra CRS2.

\begin{tabular}{|c|c|c|c|c|c|c|c|}
\hline $\mathrm{C}$ & ${ }^{13} \mathrm{C}-\delta_{\mathrm{C}}-(\alpha)$ & ${ }^{1} \mathrm{H}-\delta_{\mathrm{H}}(\alpha)$ & ${ }^{13} \mathrm{C}-\delta_{\mathrm{C}}-(\beta)$ & ${ }^{1} \mathrm{H}-\delta_{\mathrm{H}}-(\beta)$ & $\alpha$ & Amirina $\beta$ & Acetil \\
\hline 1 & 38,45 & & 38,45 & & 38,7 & 38,7 & \\
\hline 2 & 28,02 & & 28,74 & & 27,2 & 27,3 & \\
\hline 3 & 80,61 & $4,49(\mathrm{dd}, \mathrm{H})$ & 80,61 & $4,49(\mathrm{dd}, \mathrm{H})$ & 78,3 & 79,0 & 80,61 \\
\hline 4 & 38,04 & & 38,25 & & 38,7 & 38,8 & \\
\hline 5 & 55,24 & & 55,24 & & 55,2 & 55,3 & \\
\hline 6 & 18,10 & & 18,25 & & 18,3 & 18,5 & \\
\hline 7 & 32,97 & & 32,85 & & 32,9 & 32,8 & \\
\hline 8 & 40,02 & & 38,45 & & 40,0 & 38,8 & \\
\hline 9 & 47,54 & & 47,63 & & 47,7 & 47,7 & \\
\hline 10 & 36,78 & & 37,70 & & 36,9 & 37,6 & \\
\hline 11 & 23,36 & & 23,58 & & 23,3 & 23,6 & \\
\hline 12 & 124,31 & $5,12(\mathrm{dd}, \mathrm{H})$ & 121,63 & $5,12(\mathrm{dd}, \mathrm{H})$ & 124,3 & 121,8 & \\
\hline 13 & 139,19 & & 145,20 & & 139,3 & 145,1 & \\
\hline 14 & 42.05 & & 41,70 & & 42,0 & 41,8 & \\
\hline 15 & 28,74 & & 26,12 & & 28,7 & 26,2 & \\
\hline 16 & 26,92 & & 26,59 & & 26,6 & 27,0 & \\
\hline 17 & 33,74 & & 32,58 & & 33,7 & 32,5 & \\
\hline 18 & 59,04 & & 47,54 & & 58,9 & 47,4 & \\
\hline 19 & 39,64 & & 28,02 & & 39,6 & 46,9 & \\
\hline 20 & 39,60 & & 31,07 & & 39,6 & 31,1 & \\
\hline 21 & 31,24 & & 34,72 & & 31,2 & 34,8 & \\
\hline 22 & 41,53 & & 37,13 & & 41,5 & 37,2 & \\
\hline 23 & 27,98 & $1,01\left(\mathrm{~d}, \mathrm{CH}_{3}\right)$ & 28,05 & $1,01\left(\mathrm{~d}, \mathrm{CH}_{3}\right)$ & 28,1 & 28,2 & \\
\hline 24 & 15,72 & $1,11\left(\mathrm{~d}, \mathrm{CH}_{3}\right)$ & 15,54 & $1,11\left(\mathrm{~d}, \mathrm{CH}_{3}\right)$ & 15,6 & 15,5 & \\
\hline 25 & 16,16 & $0,92\left(\mathrm{~s}, \mathrm{CH}_{3}\right)$ & 15,96 & $0,89\left(\mathrm{~s}, \mathrm{CH}_{3}\right)$ & 15,6 & 15,6 & \\
\hline 26 & 16,80 & $1,05\left(\mathrm{~s}, \mathrm{CH}_{3}\right)$ & 16,85 & $0,96\left(\mathrm{~s}, \mathrm{CH}_{3}\right)$ & 16,8 & 16,9 & \\
\hline 27 & 23,21 & $1,24\left(\mathrm{~s}, \mathrm{CH}_{3}\right)$ & 25,93 & $12,4\left(\mathrm{~s}, \mathrm{CH}_{3}\right)$ & 23,3 & 26,0 & \\
\hline 28 & 28,08 & $0,79\left(\mathrm{~s}, \mathrm{CH}_{3}\right)$ & 28,39 & $0,78\left(\mathrm{~s}, \mathrm{CH}_{3}\right)$ & 28,1 & 28,4 & \\
\hline 29 & 17,48 & $0,99\left(\mathrm{~s}, \mathrm{CH}_{3}\right)$ & 33,32 & $0,95\left(\mathrm{~d}, \mathrm{CH}_{3}\right)$ & 17,4 & 33,3 & \\
\hline 30 & 21,38 & $0,81\left(\mathrm{~s}, \mathrm{CH}_{3}\right)$ & 23,68 & $0,85\left(\mathrm{~d}, \mathrm{CH}_{3}\right)$ & 21,3 & 23,7 & \\
\hline 1 , & 170,99 & & 170,99 & & & & 170,99 \\
\hline 2 & 21,38 & $2,03\left(\mathrm{~s}, \mathrm{H}_{3} \mathrm{CCO}\right)$ & 21,38 & $2,03\left(\mathrm{~s}, \mathrm{H}_{3} \mathrm{CCO}\right)$ & & & 21,38 \\
\hline
\end{tabular}


Tabela 4. Atividade antioxidante de Dorstenia multiformis Miquel pelo método de complexação do fosfomolibdênio.

\begin{tabular}{l|c|c}
\hline \multicolumn{1}{c|}{ Amostra } & $\begin{array}{c}\text { AAR\% (Ácido } \\
\text { Ascórbico) }\end{array}$ & AAR\% (Rutina) \\
\hline Extrato etanólico bruto & $21,21 \%$ & $65,30 \%$ \\
Fração hexano & $19,78 \%$ & $60,88 \%$ \\
Fração clorofórmio & $34,49 \%$ & $106,19 \%$ \\
Fração acetato de etila & $21,50 \%$ & $66,19 \%$ \\
CRS1 & $8,87 \%$ & $27,29 \%$ \\
CRS2 & $26,45 \%$ & $81,44 \%$ \\
\hline
\end{tabular}<smiles></smiles>

Figura 1. Estrutura química do psoraleno e do bergapteno.

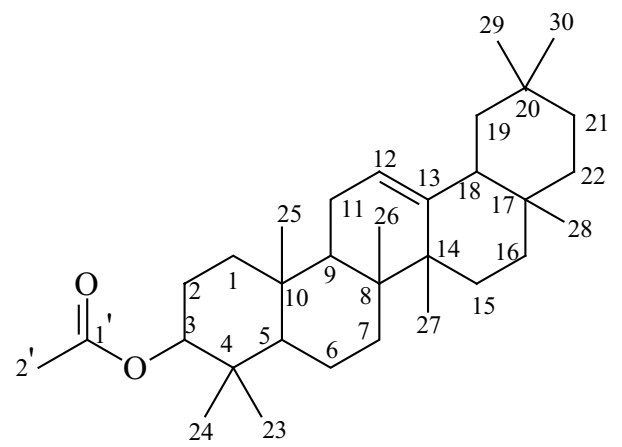

$3 \alpha-\mathrm{O}$-acetato de $\alpha$ amirina

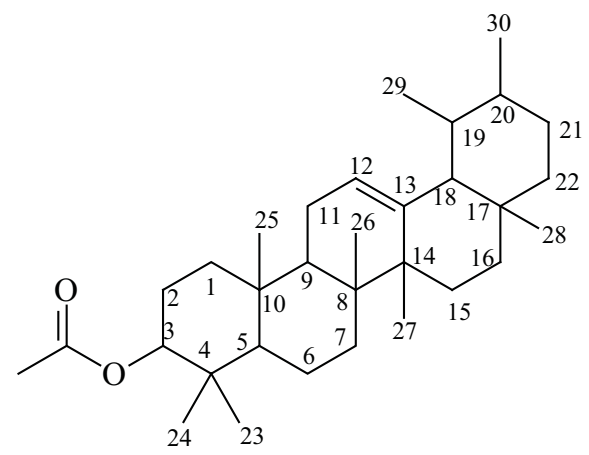

$3 \beta$-O-acetato de $\beta$ amirina

Figura 2. Estrutura química de $3 \alpha-O$-acetato de $\alpha$ amirina e $3 \beta-O$-acetato de $\beta$ amirina.
De acordo com o método descrito por Conforti et al. (2002), as amostras $(10 \mathrm{mg} / \mathrm{mL})$ foram submetidas à cromatografia em camada delgada (CCD), usando-se como fase móvel hexano: 20, acetato de etila: 30 e ácido fórmico: 2. Em seguida, as placas foram reveladas com solução a $0,2 \%(\mathrm{p} / \mathrm{v})$ de DPPH (difenil-picril-hidrazil) em etanol e fotografadas com auxílio de câmera digital.

O DPPH é um radical cromóforo que simula as espécies reativas de oxigênio (EROs); dessa forma, ele pode aceitar um elétron ou radical hidrogênio para se tornar uma molécula estável, que apenas raramente pode ser oxidada irreversivelmente. Possui coloração violeta intenso e quando aplicado em placa cromatográfica na forma de solução alcoólica, adquire coloração amarelo ouro característica em presença de substâncias com atividade antioxidante, não apresentando alteração de cor na ausência destas (Conforti et al., 2002).

\section{RESULTADOS}

\section{Identificação dos compostos CRS1 e CRS2}

A partir da análise cromatográfica em cromatografia gasosa acoplada a massa, da amostra CRS1 pode-se observar absorção em 11,19 min, indicando estrutura química do psoraleno e outro pico em 13,49 $\mathrm{min}$, indicativo da estrutura do bergapteno. Estes foram confirmados pelo espectro de $\mathrm{RMN}{ }^{13} \mathrm{C} \mathrm{e}{ }^{1} \mathrm{H}$.

Os espectros de $\mathrm{RMN}{ }^{13} \mathrm{C}$ e ${ }^{1} \mathrm{H}$ revelaram absorções que estão demonstradas nas Tabelas 2 e 3.

Comparando-se os dados do espectro aos valores relatados pela literatura (Masuda et al., 1998) para psoraleno e bergapteno (Figura 1) na Tabela 2 sugere-se que a estrutura majoritária seja o psoraleno, sendo que os sinais excedentes no espectro são coincidentes para a estrutura química do bergapteno.

No espectro de RMN ${ }^{1} \mathrm{H}$ para o composto CRS2 observa-se o perfil característico de triterpenos, conforme Tabela 3. A presença de 10 metilas singleto e seis metilas duplete no espectro de próton é confirmada no carbono-13, sugerindo um esqueleto pentacíclo derivado do ursano e lanostano contendo uma ligação dupla trisubstituída definida pelos sinais de próton em $5,12 \mathrm{ppm} \mathrm{H}$, e carbono em 124,1 e $121,7 \mathrm{ppm} \mathrm{CH}$ e 139,4 e $145,1 \mathrm{ppm}$ para C. O duplo duplete centrado em 4,49 ppm para um próton carbinólico correlacionado ao $\mathrm{CH}$ em 80,61 indicam a presença de $3 \beta$-acetato. A comparação dos deslocamentos químicos de carbono-13 com dados da literatura (Barreto et al., 1998; Gallegos \& Roque, 1990; Bandeira et al., 2007) permitiu identificar os triterpenos $3 \beta-O$-acetato de $\beta$-amirina e $3 \alpha-O$-acetato de $\alpha$-amirina (Figura 2).

\section{Avaliação da atividade antioxidante}

Os resultados da avaliação da atividade 


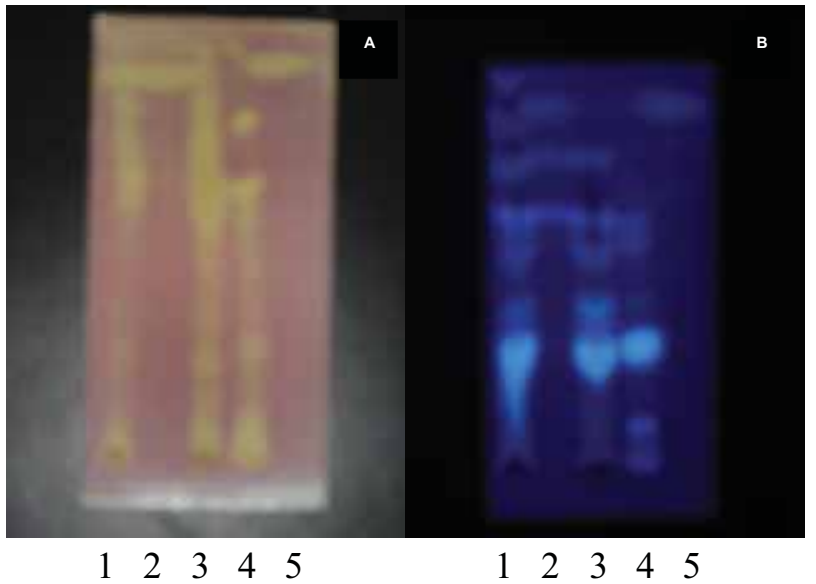

Notas: 1. Pontos 1, 2, 3, 4 e 5: extrato bruto etanólico, fração hexano, fração clorofórmio, fração acetato de etila e CRS1, respectivamente.

2. Figura A - com revelador DPPH e figura B - visualização em câmara de UV.

Figura 3. Atividade antioxidante pelo ensaio de cromatografia em camada delgada.

antioxidante pelo método de complexação do fosfomolibdênio estão apresentados na Tabela 4 e os resultados da avaliação da atividade antioxidante por cromatografia de camada delgada na Figura 3.

\section{DISCUSSÃO}

De acordo com os resultados apresentados, todas as amostras testadas (extrato etanólico bruto, fração hexano, fração clorofórmio, fração acetato de etila e amostras CRS1 e CRS2) demonstraram atividade antioxidante por redução do complexo fosfomolibdênio, ao adquirir a coloração verde característica. Sugere-se que a atividade antioxidante observada para o extrato bruto e as frações não seja devida aos compostos psoraleno e bergapteno (presentes na amostra CRS1), uma vez que os valores de atividade antioxidante total, obtidos para CRS1 encontram-se significativamente abaixo daqueles obtidos para as demais amostras testadas, atribuindo-se uma baixa atividade antioxidante para esses compostos cumarínicos, por comparação com os padrões utilizados (vitamina $\mathrm{C}$ e rutina).

Dessa forma, considerando-se que o extrato etanólico bruto e as frações obtidas do extrato tratamse de misturas complexas de componentes químicos, pode-se supor que a atividade antioxidante mais pronunciada, observada para essas amostras, em relação à amostra CRS1, possa ser devida a um sinergismo dos componentes presentes nas referidas amostras com as cumarinas psoraleno e bergapteno. Ainda, pode-se sugerir a presença dos compostos terpenóides no extrato bruto etanólico e frações, os quais estão presentes na amostra CRS2, uma vez que tais compostos apresentaram uma atividade antioxidante relativa significativa, quando comparado aos padrões: vitamina $\mathrm{C}$ e rutina.

$\mathrm{Na}$ avaliação da atividade antioxidante considere-se que a fração clorofórmio apresentou atividade antioxidante relativa superior, quando comparada ao padrão de atividade antioxidante rutina no método de complexação do fosfomolibdênio.

Avaliando-se a atividade antioxidante por cromatografia de camada delgada, observa-se na figura 3 presença de um grande número de substâncias com atividade antioxidante nas amostras testadas, especialmente na fração clorofórmio, confirmando o resultado obtido de atividade antioxidante significativa para essa amostra com o reativo fosfomolibdênio.

Dessa forma, a fração clorofórmio pode ser considerada um antioxidante em potencial, com possibilidade de aplicação na terapêutica, e inclusive com possibilidade de separação de princípios ativos.

\section{AGRADECIMENTOS}

A Indústria "As Ervas Curam" pelo fornecimento do material vegetal.

\section{REFERÊNCIAS}

Abegaz BM, Ngadjui BT, Dongo E, Ngameni B, Nindi MN, Bezabih M 2002. Chalcones and other constituents of Dorstenia prorepens and Dorstenia zenkeri. Phytochemistry 59: 877-883.

Agra MF, França PF, Barbosa-Filho JM 2007. Synopsis of the plants known as medicinal and poisonous in Northeast of Brazil. Rev Bras Farmacogn 17: 114-140.

Amaral FMM, Ribeiro MNS, Barbosa-Filho JM, Reis AS, Nascimento FRF, Macedo RO 2006. Plants and chemical constituents with giardicidal activity. Rev Bras Farmacogn 16(Supl.): 696-720.

Bandeira PN, Lemos TLG, Costa SMO, Santos HS 2007. Obtenção de derivados da mistura triterpenoídica $\alpha$ e $\beta$-amirina. Rev Bras Farmacogn 17: 204-208.

Bauer L, Noll IB 1986. Furanocumarinas de Dorstenia brasiliensis Lam. Caderno de Farmácia 2: 163-170.

Barreto AS, Carvalho MG, Nery IA, Gonzaga L, Kaplan MAC 1998. Chemical constituints from Himatanthus articulate. J Braz Chem Soc 9: 430-434.

Caceres A, Rastrelli L, De Simone F, De Martino G, Saturnino C, Saturnino P, Aquino R 2001. Furanocumarins from the aerial parts of Dorstenia contrajerva. Fitoterapia 72: 376-381.

Cardoso CAL, Vilegas W, Barison A., Honda NK 2002. Simultaneous determination of furanocumarins in infusions and decoctions from "Carapiá" (Dorstenia species) by high-performance liquid chromatography. J Agr Food Chem 50: 1465-1469.

Carvalho JLS 2001. Contribuição ao estudo fitoquímico $e$ analítico de Nasturtium officinale $R$. BR., Brassicaceae. Curitiba. Dissertação de Mestrado em Ciências Farmacêuticas - Setor de Ciências da Saúde, Universidade Federal do Paraná.

Casagrande C, Ronchetti F, Russo G 1974. Structure of syriogenin. Tetrahedron 30: 3587-3589. 
Conforti F, Statti GA, Tundis R, Menichini F, Houghton P 2002. Antioxidant activity of methanolic extract of Hypericum triquetrifoium, Turra aerial part. Fitoterapia 73: 479-483.

Cronquist A 1981. An integrated system of classification of floring plants. New York: Columbia University Press.

Dimo T, Rakotonirina A, Tan PV, Dongo E, Dongmo AB, Kamtchouing P, Azay J, Abegaz BM, Cros G, Ngadjui TB 2001. Antihypertensive effects of Dorstenia psilurus extract in fructose-fed hyperinsulinemic, hypertensive rats. Phytomedicine 8: 101-106.

Falcão HS, Lima IO, Santos VL, Dantas HF, Diniz MFFM, Barbosa-Filho JM, Batista LM 2005. Review of the plants with anti-inflammatory activity studied in Brazil. Rev Bras Farmacogn 15: 381-391.

Gallegos RS, Roque NF 1990. Análise de misturas de triterpenos por RMN de ${ }^{13}$ C. Quim Nova 13: 278-281.

Iwu MM, Jackson JE, Tally JD, Klayman DL 1992. Evaluation of plant extracts for antileishmanial activity using a mechanism-based radiorespirometric microtechnique (RAM). Planta Med 58: 436-441.

Joly AB 1975. Introdução à Taxonomia Vegetal. Câmara Brasileira do Livro.

Lopes D, Oliveira RR, Kaplan MAC, Lage CS, Leitão AC 2001. Photosensitization and mutation induced in Escherichia coli and Saccharomyces cerevisiae strains by dorstenin, a psoralen analog isolated from Dorstenia bahiensis. Planta Med 67: 820-824.

Masuda T, Takasugi M, Anetai M 1998. Psoralen and other linear furanocoumarins as phytoalexins in Glehnia littoralis. Phytochemistry 47: 13-16.

Ngadjui BT, Dongo E, Abegaz BM, Fotso S, Tamboue H 2002. Dinklagins A, B and C: threee prenylated flavonoids and other constituents from the twigs of Dorstenia dinklagins. Phytochemistry 61: 99-104.

Prieto P, Pineda M, Aguilar M 1999. Spectrophotometric quantitation of antioxidant capacity through the formation of a phosphomolybdenium complex:specific aplication to the determination of vitamin E. Anal Biochem 269: 337-341.

Ruppelt BM, Pereira EF, Gonçalves LC, Pereira NA 1991. Pharmacological screening of plants recommended by folk medicine as anti-snake venom - I. Analgesic and anti-inflammatory activities. Mem I Oswaldo Cruz 86: 203-205.

Tsopmo A, Tene M, Kamnaing P, Ngnokam D, Ayafor JF, Sterner O 1998. Geranylated flavonoids from Dorstenia poinsettifolia. Phytochemistry 48: 345-348.

Vilegas W, Vilegas JHY, Pozetti GL 1992. Cromatografia de permeação em gel das furanocumarinas de Dorstenia heringeri Car. \& Val. Revista de Ciências Farmacêuticas 14: 133-138.

Vilegas JHY, Lancas FM, Vilegas W, Pozetti GL 1997. Further triterpenes, steroids and furanocumarins from Brazilian medicinal plants of Dorstenia genus (Moraceae). J Braz Chem Soc 8: 529-535.

Youngson R 1996. Como combater os Radicais Livres.1996. Rio de Janeiro: Editora Campus. 